\title{
Review
}

Clinical

nephron

Practice

\section{Biomarkers in Acute Kidney Injury: Are We Ready for Prime Time?}

\author{
Prasad Devarajan ${ }^{a}$ Patrick Murray ${ }^{b}$ \\ ${ }^{a}$ Nephrology and Hypertension, Cincinnati Children's Hospital Medical Center, University of Cincinnati College \\ of Medicine, Cincinnati, Ohio, USA; ${ }^{b}$ School of Medicine and Medical Science, Health Sciences Centre, University \\ College Dublin, Dublin, Ireland
}

\section{Key Words}

Biomarker · Acute kidney injury · Kidney damage · Kidney function

\begin{abstract}
Novel biomarkers are required to improve the timely detection of early acute kidney injury (AKI) and to improve the differential diagnosis, prognostic assessment, and management of AKI cases. It is anticipated that novel biomarkers of early structural AKI ('acute kidney damage') will provide critical diagnostic and prognostic stratification and complement functional markers such as serum creatinine. Further studies are required to conclusively demonstrate the association between early kidney damage biomarkers and clinical outcomes, both with and independently of functional markers, and to discern whether or not randomization to a treatment for AKI based on high structural/damage biomarker levels results in an improvement in kidney function and clinical outcomes.

(c) 2014 S. Karger AG, Basel
\end{abstract}

Changing Paradigms in Acute Kidney Injury: From Mechanisms to Management - Proceedings of the 5th Annual UAB-UCSD O'Brien Center Symposium (San Diego, Calif., USA, March 4, 2014).

\section{KARGER}

E-Mail karger@karger.com

www.karger.com/nec
Our long-standing reliance on serum creatinine measurements has clearly hindered progress in acute kidney injury (AKI), the incidence of which is increasing globally [1]. This condition accounts for $3-5 \%$ of admissions in general hospitals, with serious adverse outcomes that have not changed significantly in the past several decades. Serum creatinine, the current standard for measurement of kidney function and dysfunction, is a flawed AKI marker for many reasons. First, a single measurement of serum creatinine cannot distinguish true structural (intrinsic) AKI (with acute renal tubular damage) from chronic kidney disease or from functional, and potentially hemodynamically reversible, AKI ('prerenal azotemia') [2]. It is critical to make these distinctions, since the management of each is distinct and mismanagement is deleterious. Second, creatinine levels are dramatically influenced by several nonrenal factors such as age, gender, muscle mass, muscle metabolism, medication use, hydration status, and nutrition status. Third, an increase in serum creatinine is delayed in AKI due to a number of factors, including: significant renal functional reserve (in many patients, but not necessarily in those with chronic kidney disease), the obligatory time required for the accumulation of circulating creatinine before a new steadystate serum concentration is reached, and the masking and further delay of serum creatinine increments by a 
positive fluid balance and hemodilution [3]. Novel biomarkers to improve the detection of early AKI and provision of early interventions are therefore a subject of intense contemporary interest [4].

An ideal AKI biomarker should fulfill the characteristics listed in table 1 . The majority of novel AKI biomarkers described thus far have been measured in urine. Urinary diagnostics have advantages, including the noninvasive nature of sample collection, the reduced number of interfering proteins, and the increased specificity for kidney injury. However, disadvantages include the lack of samples from patients with severe oliguria, and potential changes in urinary biomarker concentrations induced by the hydration status and diuretic therapy. A commonly employed correction factor for urinary dilution is to express urinary biomarkers adjusted for urinary creatinine concentration in research studies. However, this correction may be inaccurate in the situation of AKI because: (a) creatinine production may be reduced in some forms of AKI, and (b) both plasma and urine creatinine kinetics are significantly altered in the early phases of AKI [5]. Plasma-based diagnostics have revolutionized many facets of medicine, in part due to the easy accessibility of samples. However, plasma biomarkers may be confounded by extrarenal sources as well as by subclinical changes in renal elimination. Thus, in the case of AKI, it is important and ideal to develop both urinary and plasma biomarkers.

Over the past decade, the application of unbiased functional genomic and proteomic technologies has identified a number of novel AKI biomarkers. Many represent the early stress response of the kidney to AKI, induced in the kidney tubules during the early damage phase, and serendipitously appear in the urine or plasma well before a change in serum creatinine is detected [6]. Many are being developed and validated as early noninvasive biomarkers for the prediction of AKI and its clinical outcomes in humans. This is a rapidly evolving field, and the current status of some of the most promising examples is shown in table 2 . The list is certainly not exhaustive, since new markers are being continually discovered and compared to the ones listed [7].

The most widely studied and validated early biomarker of AKI in children is neutrophil gelatinase-associated lipocalin (NGAL) [8]. Following cardiac surgery, plasma or urine NGAL measurements in over 6,600 patients were predictive of AKI and its severity, with an overall area under the receiver operating characteristic curve of $0.8-$ 0.82 . Similar results were obtained in over 6,000 critically ill patients. In 972 patients undergoing kidney transplantation, NGAL measurements predicted a delayed graft
Table 1. Desirable characteristics of AKI biomarkers

\section{Biomarker property}

Minimally invasive measurement techniques using urine or blood

Rapid and inexpensive to measure

Results are available while damage is limitable

Amendable to clinical assay platforms

Sensitive to establish an early diagnosis

High gradient to allow risk stratification

Specific to intrinsic AKI (differentiate from prerenal AKI and CKD)

Increase is proportional to the degree of damage

Biologic plausibility - marker associated with a known mechanism

Identify the primary location of injury within the kidney

Results predict clinical outcomes

Results predict the response to therapies

Results expedite the drug development process

$\mathrm{CKD}=$ Chronic kidney disease.

function with an overall area under the receiver operating characteristic curve of 0.88 . In all three settings, NGAL significantly improved the prediction of AKI risk over the clinical model alone [8]. A large multicenter pooled analysis of existing NGAL studies was recently published, confirming the utility of this marker for the early diagnosis of AKI and its adverse outcomes [9].

A recent study examined a combination of biomarkers following cardiac surgery [10]. Urinary NGAL was increased in AKI patients within $2 \mathrm{~h}$ of bypass initiation, urine interleukin-18 (IL-18) and liver-type fatty acid binding protein (L-FABP) were increased within $6 \mathrm{~h}$, and urine kidney injury molecule-1 (KIM-1) increased at the 12-hour time point. All markers correlated with AKI severity and clinical outcomes and improved the risk prediction for AKI over clinical models. Thus, they represent temporally sequential markers, and a panel of such biomarkers may therefore help establish the timing of the injury and plan appropriate therapies.

The concept of outcomes in 'biomarker-positive, creatinine-negative' patients has recently been explored [9, 11]. Two studies enrolling about 4,000 cardiac surgical, critically ill, or emergency department patients grouped subjects according to their NGAL and serum creatinine status. Both studies found that measurement of NGAL complemented the information obtained by measurement of serum creatinine levels in establishing the diagnosis of AKI and predicting the prognosis. A substantial 
Table 2. Promising urinary biomarkers for the prediction of AKI and its outcomes in various clinical settings

\begin{tabular}{|c|c|c|c|c|c|}
\hline Biomarker & Source & Function & Cardiac surgery & Kidney transplant & ICU/ED \\
\hline IL-18 & Proximal tubule & $\begin{array}{l}\text { Promotes tubule cell } \\
\text { apoptosis and necrosis }\end{array}$ & $\begin{array}{l}6 \mathrm{~h} \text { after } \mathrm{CPB} \\
2 \text { days before AKI } \\
\text { Predicts AKI severity, } \\
\text { dialysis, and death }\end{array}$ & $\begin{array}{l}6 \mathrm{~h} \text { after transplant } \\
2-3 \text { days before DGF } \\
\text { Predicts long-term graft loss }\end{array}$ & $\begin{array}{l}\text { On admission } \\
1-2 \text { days before AKI } \\
\text { Predicts AKI severity, dialysis, } \\
\text { and death }\end{array}$ \\
\hline L-FABP & Proximal tubule & $\begin{array}{l}\text { Antioxidant, suppresses } \\
\text { tubule-interstitial damage }\end{array}$ & $\begin{array}{l}6 \mathrm{~h} \text { after } \mathrm{CPB} \\
2 \text { days before AKI } \\
\text { Not tested for outcomes }\end{array}$ & Not tested & $\begin{array}{l}\text { On admission } \\
1-2 \text { days before AKI } \\
\text { Predicts AKI severity, dialysis, } \\
\text { and death }\end{array}$ \\
\hline KIM-1 & Proximal tubule & $\begin{array}{l}\text { Promotes epithelial } \\
\text { regeneration, regulates } \\
\text { apoptosis }\end{array}$ & $\begin{array}{l}12 \mathrm{~h} \text { after } \mathrm{CPB} \\
1 \text { day before AKI } \\
\text { Not tested for outcomes }\end{array}$ & Not tested & $\begin{array}{l}\text { On admission } \\
1-2 \text { days before AKI } \\
\text { Predicts AKI severity, dialysis, } \\
\text { and death }\end{array}$ \\
\hline
\end{tabular}

AKI is defined as AKIN stage I or greater. The times shown (in h or days) are the earliest time points when the biomarker becomes significantly increased from baseline. $\mathrm{CPB}=$ Cardiopulmonary bypass; $\mathrm{DGF}=$ delayed graft function; ICU = intensive care unit; $\mathrm{ED}=$ emergency department.

proportion of patients (about 20\%) had elevated NGAL levels even in the absence of loss of renal excretory function. This previously undetectable condition ('subclinical AKI') was associated with a 2- to 3-fold increased risk of death or the need for RRT compared to patients in whom levels of serum creatinine were not elevated. Notably, even in patients with a significant loss of renal function, measurement of tubule damage biomarker levels still added prognostic information, as patients with increased levels of NGAL and serum creatinine levels displayed by far the worst prognoses.

It is anticipated that novel biomarkers of early structural AKI ('acute kidney damage') will provide critical diagnostic and prognostic stratification and will complement functional markers such as serum creatinine [2]. Are they ready for prime time? The eminent speakers in this session tackled that very question. As can be gleaned from their individual presentations, AKI biomarkers are indeed ready for more rigorous testing and validation. Dr. Endre reviewed a number of limitations of the AKI biomarker studies performed to date, including the lack of a true 'gold standard' in this field, and suggested approaches to their further validation and implementation [12]. Dr. Kellum reviewed emerging candidate biomarkers of recovery following AKI [13]. Dr. Parikh reviewed the significant discoveries (including relationships with short- and long-term clinical outcomes) of the TRIBE consortium from their prospective, multicenter study of AKI biomarkers in patients undergoing cardiac surgery [14]. Dr. Wendon and Dr. Maisel discussed potential applications of new AKI damage biomarkers along with existing tools for the evaluation and management of patients with hepatorenal [15] and cardiorenal [16] syndromes, respectively. This should include large enough future studies that demonstrate: (a) the association between early structural biomarkers and hard outcomes such as dialysis, cardiovascular events, and death, both with and independent of functional markers, and (b) that randomization to a treatment for AKI based on high structural/damage biomarker levels results in an improvement in kidney function and amelioration of adverse clinical outcomes. Successful demonstration of these two types of studies will truly launch AKI biomarkers for routine clinical application.

\section{Disclosure Statement}

P.D. is a coinventor on patents submitted for the use of NGAL as a biomarker of kidney injury.

P.M. has received research funding from Abbott, Alere, and Argutus/EKF Diagnostics and served as a consultant or advisory board member for Argutus/EKF Diagnostics, FAST Biomedical, and Abbott. 


\section{References}

$>1$ Lameire NH, Bagga A, Cruz D, et al: Acute kidney injury: an increasing global concern. Lancet 2013;382:170-179.

$\checkmark 2$ Murray PT, Mehta RL, Shaw A, et al: Potential use of biomarkers in acute kidney injury: report and summary of recommendations from the 10th Acute Dialysis Quality Initiative consensus conference. Kidney Int 2014;85:513521.

$\checkmark 3$ Macedo E, Bouchard J, Soroko SH, et al: Fluid accumulation, recognition and staging of acute kidney injury in critically-ill patients. Crit Care 2010;14:R82.

-4 Devarajan P: Neutrophil gelatinase-associated lipocalin: a promising biomarker for human acute kidney injury. Biomark Med 2010; 4:265-280.

5 Waikar SS, Bonventre JV: Creatinine kinetics and the definition of acute kidney injury. Am Soc Nephrol 2009;20:672-679.

6 Devarajan P: Biomarkers for the early detection of acute kidney injury. Curr Opin Pediatr 2011;23:194-200.
7 Kashani K, Al-Khafaji A, Ardiles T, et al: Discovery and validation of cell cycle arrest biomarkers in human acute kidney injury. Crit Care 2013;17:R25.

$>8$ Haase-Fielitz A, Haase M, Devarajan P: Neutrophil gelatinase-associated lipocalin as a biomarker of acute kidney injury: a critical evaluation of current status. Ann Clin Biochem 2014;51:335-351.

-9 Haase M, Devarajan P, Haase-Fielitz A, et al: The outcome of neutrophil gelatinase-associated lipocalin-positive subclinical acute kidney injury: a multicenter pooled analysis of prospective studies. J Am Coll Cardiol 2011; 57:1752-1761.

10 Krawczeski CD, Goldstein SL, Woo JG, et al: Temporal relationship and predictive value of urinary acute kidney injury biomarkers after pediatric cardiopulmonary bypass. J Am Coll Cardiol 2011;58:2301-2309.

11 Nickolas TL, Schmidt-Ott KM, Canetta P, et al: Diagnostic and prognostic stratification in the emergency department using urinary biomarkers of nephron damage: a multicenter prospective cohort study. J Am Coll Cardiol 2012;59:246-255

-12 Endre ZH, Pickering JW, Walker RJ, et al: Improved performance of urinary biomarkers of acute kidney injury in the critically ill by stratification for injury duration and baseline renal function. Kidney Int 2011;79:1119-1130.
3 Murugan R, Wen X, Shah N, et al; for the Biological Markers of Recovery of Kidney (BioMaRK) Study Investigators: Plasma inflammatory and apoptosis markers are associated with dialysis dependence and death among critically patients receiving renal replacement therapy. Nephrol Dial Transplant 2014, Epub ahead of print.

14 Coca SG, Garg AX, Thiessen-Philbrook H, et al; for the TRIBE-AKI Consortium: Urinary biomarkers of AKI and mortality 3 years after cardiac surgery. J Am Soc Nephrol 2013, Epub ahead of print.

15 Slack A, Yeoman A, Wendon J: Renal dysfunction in chronic liver disease. Crit Care 2010;14:214.

16 Maisel AS, Mueller C, Fitzgerald R, et al: Prognostic utility of plasma neutrophil gelatinaseassociated lipocalin in patients with acute heart failure: the NGAL EvaLuation Along with B-type NaTriuretic Peptide in acutely decompensated heart failure (GALLANT) trial. Eur J Heart Fail 2011;13:846-851. 